\title{
A System Architecture for Low Bit Rate Traffic Aggregation in Control Applications
}

\author{
J.M.Cabral ${ }^{1}$, J.G.Rocha ${ }^{1}$, J.E.Neves ${ }^{1}$ and J.Ruela ${ }^{2}$ \\ ${ }^{1}$ Industrial Electronics Department, University of Minho, Campus de Azurém, 4800-058 Guimarães, Portugal \\ ${ }^{2}$ School of Engineering, University of Porto (FEUP-DEEC) / INESC Porto, Rua Dr. Roberto Frias, 4200-465 Porto, Portugal \\ jose.cabral@dei.uminho.pt
}

\begin{abstract}
This paper describes the results of research work aimed at providing an efficient solution to the aggregation of low bit rate traffic in control applications. It includes the proposal and analysis of an architecture that integrates Terminal Adapters and a Concentrator connected to a remote Control Application through a communication network. The ATM technology was selected to implement the proposed system due to its properties of multiplexing efficiency and control of packet delay. Scheduling Algorithms for the Terminal Adapter and Concentrator have been developed in order to satisfy two main goals: to guarantee committed delays for time sensitive services, and to increase the network transmission efficiency. The results, obtained by simulation, show that the system performance complies with the requirements of the majority of control applications.
\end{abstract}

\section{INTRODUCTION}

The technological evolution at the microelectronic level has led to the development of new devices (e.g. sensors, actuators, data acquisition systems) at very low prices. As a consequence, the number of distributed control applications, which involve the interconnection of a great variety of equipment, is growing in areas such as agriculture, cattle units, domotics, industrial control and automotive industry. The communication between such devices can be characterized, in general, by producing individual flows with very low bit rate and requiring low transmission delays. This kind of traffic is usually supported by specific networks that are usually called Fieldbuses; CAN and Profibus are examples of Fieldbus technologies. They have many limitations, mainly in aspects related with the integration of services and systems, bandwidth and coverage area.

On the other hand, until recently communications networks have been optimized to support specific services (e.g. transmission of voice, video or data files), thus requiring some form of adaptation to support other types of services. The current trend towards integration of services in the same network is usually associated with the need to support differentiated Quality of Service (QoS). Moreover, adapting low bit rate services in such networks is concomitant with the control of time delay in assembling and scheduling packets; these aspects have a significant impact on QoS.

To solve these problems a modular system architecture was studied and specified. It allows multiplexing individual flows, generated by low bit rate services, into a single aggregate flow.

The main objective of this system is to aggregate low bit rate traffic, usually associated to Fieldbuses, for transmission over a communication network. ATM (Asynchronous Transfer Mode) was the selected technology due to its capability of multiplexing in an efficient way a large number of data flows, while supporting different delay requirements [1].

The paper is organized as follows. Section II analyses the problem of aggregating low bit rate traffic in control applications. Section III describes the system architecture and section IV specifies the scheduling algorithms used to perform traffic aggregation. Section $\mathrm{V}$ describes simulation results and, finally, in section VI some conclusions are presented.

\section{Aggregation of Low Bit RAte TrafFic In CONTROL APPLICATIONS}

Usually, a sensor network is composed of a large number of small devices, whose main objective is to detect and transmit some physical characteristic of the environment [2]. These components or nodes can be used in an efficient way, even in the case where their number is in the order of thousands, with a single common objective. A control system may integrate a large number of sensors, actuators and respective control entities. Therefore, even when the communication between each pair of devices is characterised by a low bit rate and requires moderate or small transmission delays, the aggregate bit rate to be supported by the network can reach very high values and time delays may become unacceptable, if not properly controlled.

\section{A. Application scenarios}

There are many applications where distributed data acquisition and control systems become necessary to cover large and dispersed geographical areas. Examples are:

- Agricultural greenhouses,

- Cattle units,

- Textile industry,

- Meteorological stations,

- Fire detection systems in forests.

Sensors can also be used to monitor places with difficult access or dangerous environments, such as ocean deep, neighbourhoods of volcanic activity, enemy territories, disaster areas and nuclear activity places. They can also be used in interactive tasks, such as finding and detonating explosives, or locating survivors in natural disaster areas.

In the examples described above, the information is collected in the places where the phenomena occur, and are translated to measurable quantities (e.g. electrical voltage or 
current), being then converted to a digital format to be further processed and conveyed to a remote place through a communication network.

\section{B. Traffic Classes}

At this point it is necessary to characterize the way different traffic flows, generated by different devices and systems, will be treated by a traffic aggregation system. In this way, the different performance levels supported by the control system may be associated with three traffic classes:

- Maximum Delay (MD)

The flows associated with this traffic class need a maximum and well-defined time delay guarantee between the sensing device and the control application, and between the control application and the acting device.

- $\operatorname{Data}(D)$

In this traffic class, the delivery of the information has not critical delay requirements. The only requirement is that all the data must be delivered without losses, which may require a reliable end-to-end transport protocol to recover from network losses.

- Minimum Effort (ME)

This traffic class can be used when occasional loss or high delay in the information delivery does not affect the control process.

\section{Transmission delays}

The time required for data transmission along a network includes two components: the delay in processing data packets in terminal and network devices (e.g. packetization and queuing delays) and the propagation delay [3].

The value of $400 \mathrm{~ms}$ was considered a meaningful limit for network planning purposes, where speech transmission performance was the focus. Table I shows the acceptable time limits, in the case of unidirectional transmission with echo adequately controlled, according to the ITU-T G.114 recommendation [3].

TABLE I

END-TO-END TRANSMISSION TIME LIMITS [3]

\begin{tabular}{|c|c|}
\hline Delay & Acceptability \\
\hline $0-150 \mathrm{~ms}$ & Acceptable in the majority of applications \\
\hline $150-400 \mathrm{~ms}$ & Acceptable when the impact in the application allows it \\
\hline$>400 \mathrm{~ms}$ & Unacceptable \\
\hline
\end{tabular}

\section{SYSTEM ARCHITECTURE}

The general architecture of the system proposed in this paper is composed of one or more Terminal Adapters and a Concentrator connected to a Control Application, through a communications network, as shown in Fig. 1 [4].

The communication network can be an ATM network or another system with the ability to carry, in a transparent way, ATM cells. The choice of ATM to support traffic aggregation in the Terminal Adapters and Concentrator is independent of the network transmission environment and thus can be adapted to any particular network technology.

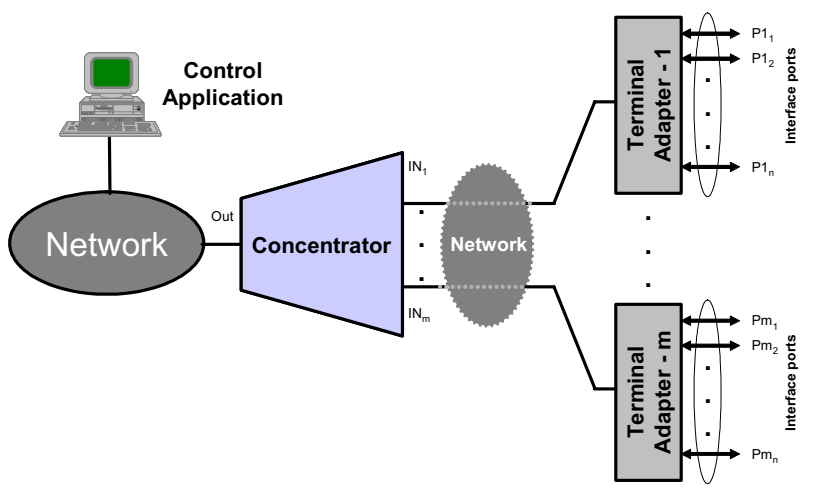

Fig. 1. Block diagram of the system developed to aggregate low bit-rate traffic in control applications.

The Terminal Adapter and Concentrator are modules that allow the optimization of the bandwidth usage by aggregating low bit rate flows from the Fieldbus devices. This aggregation can be extended to other types of traffic, generated by other services, thus leading to an even better use of network resources. These modules may also guarantee the transport of services with stringent delay requirements, which is achieved by means of scheduling algorithms, as discussed in section IV.

The Terminal Adapter allows the connection of all Fieldbus devices to the control application. Each Terminal Adapter port supports the connection of a single device (e.g. sensor, actuator, data acquisition system) or a device that is able to aggregate traffic (e.g. actuator panel, battery of integrated sensors, keyboard). Depending on the QoS requirements of each service (i.e. interface port of the Terminal Adapter) the goal of traffic aggregation is to optimize the bandwidth usage without exceeding the maximum acceptable delay specified for each service (if any). Traffic aggregation (multiplexing) will be supported by CPS (Convergence Packet Sub-layer) packets, in conformity with AAL-2 (ATM Adaptation Layer - Type 2) protocol [5] and by traffic scheduling algorithms, which will be specified and analyzed in the next section.

The identification of the traffic sources can be made directly using the CID (Channel Identifier) field of the header of the CPS packets. If the capacity provided by this mechanism were not enough, it is possible to use the ATM layer identifiers: VCI (Virtual Channel Identifier) and VPI (Virtual Path Identifier).

The Concentrator performs aggregation of traffic from Terminal Adapters, acting as a second level multiplexer. Its main objective is to increase network transmission efficiency; moreover, it implements a priority mechanism, to allow traffic scheduling based on different delay requirements.

The Concentrator also uses CPS packets as the elementary multiplexing structure. It first decapsulates the packets coming from each Terminal Adapter and then makes a new scheduling decision, so that packets with lower delay requirements are served with higher priority. Notice that this module is not necessary in a structure with a single Terminal Adapter. In this 
case, the Terminal Adapter and the Control Application are directly connected through the network.

The Control Application is the module that allows the management of the communication process among all the elements of the data acquisition and control systems. It consists of a workstation, connected to a communications network, where a set of programs execute specific tasks and where information produced by the connected devices is collected and processed. According to the application, it could also be necessary to send information, in the reverse direction, to other devices connected to Terminal Adapters. Although a large number of scenarios may be envisaged, it is important that the proposed solution is capable of guaranteeing the adequate information transfer, not only between the data acquisition and control system elements, but also between other systems that share the network, with the required QoS.

\section{A. Terminal Adapter}

The Terminal Adapter can be considered as a set of sending and receiving state machines that work in an independent way. At the sending side it is necessary to multiplex traffic flows from various sources (e.g. sensors) and with different delay requirements, into a single flow, guaranteeing the QoS and maximizing the transmission efficiency. In the receiving side it is necessary to demultiplex the channels, in order to deliver the individual flows to the actuators of the control system. Fig. 2 shows the Terminal Adapter functional structure.

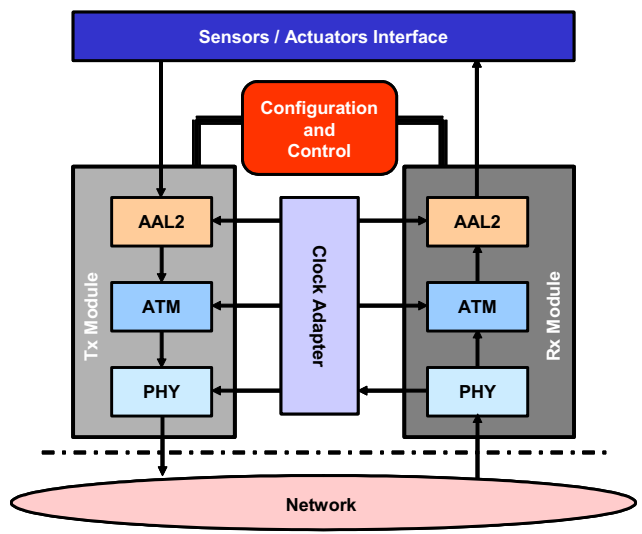

Fig. 2. Terminal Adapter functional structure.

The proposed solution for the Terminal Adapter is based on ATM technology. It establishes the interface between the network and sensors/actuators and allows solving the identification problems of these devices by the control application. This identification is made through virtual channel and virtual path identifiers (VCI/VPI) used at this interface, and through the multiplexing structure of the adaptation layer (CID/AAL-2). The details related with the data format of this protocol are described in ITU-T Recommendation I.363.2 [5]. The CID field of AAL-2 specification identifies the channel that is being used. The channels are numbered from 8 to 255 , once the recommendation reserves the values between 0 and 7 to other functions. The LI field indicates the length of the information field (CPS-INFO) in octets. For each channel, the value of LI indicates how many octets have been read from the FIFO of the corresponding input. This value can vary, for each channel, at each reading process, as a function of the number of octets available in the input FIFO. According to [5], the UUI field can serve two functions: to carry specific information in a transparent way, through the CPS sub-layer, and to distinguish between a SSCS entity (in case its value is between 0 and 27) and the management layer. In the context of this work, the UUI field is used to address the Terminal Adapter and to implement a mechanism for identification of the traffic class associated with the flow carried by the CPS packet.

As soon as they are created, CPS packets are placed in an intermediate FIFO. Here, the scheduling of the input flows had already been made. These packets have already defined the channel identifier (CID), the identification of the Terminal Adapter where they come from (UUI) and the priority associated with the traffic class assigned at the input (UUI) by the configuration module. CPS packets of possibly different sizes are concatenated and placed in blocks of 48 octets (ATM_SDUs), which are encapsulated in ATM cells.

In order to organize the transmission scheduling, each packet has a time-stamp associated to allow controlling the delay in the Terminal Adapter. Since some input traffic can have stringent delay requirements, a packet cannot wait more than a well-defined time interval. Thus, if the value of this time interval is too low, the ATM cells will be only partially filled, once the arrival ratio of CPS packets is low compared to the multiplexing clock. Otherwise, ATM cells will be totally filled but the packetization delay will increase.

\section{B. Concentrator}

The Concentrator, like the Terminal Adapter, can be considered as a set of concentration and expansion state machines that work in an independent way. Fig. 3 illustrates the working principle of the Concentrator.

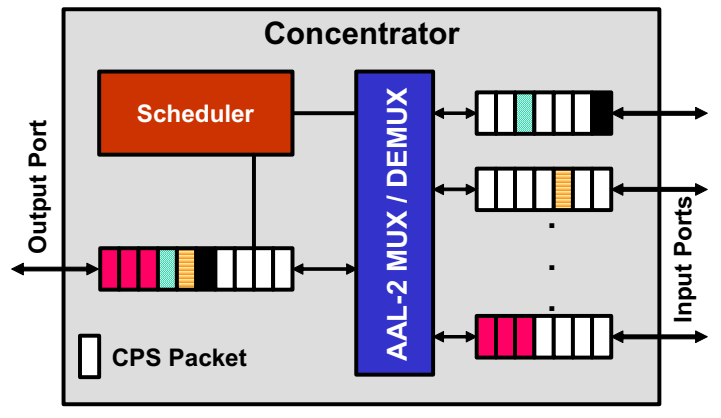

Fig. 3. Working principle of the Concentrator

The concentration unit multiplexes the CPS packets coming from the Terminal Adapters into a single aggregate flow, maximizing transmission efficiency and the expansion unit demultiplexes the CPS packets into separate flows, to be delivered to the corresponding Terminal Adapters.

The concentration unit needs to recover the CPS packet structure of the input flows, in order to multiplex them again, thus optimizing filling ratio of the ATM cells and, as a consequence, increasing the network transmission efficiency. 
Like in the Terminal Adapter, the multiplexing of flows is made by means of a scheduling algorithm based on the class of traffic associated with each flow.

The main advantage of using the Concentrator is to increase the transmission efficiency, especially when some Terminal Adapters are not making full use of their bandwidth. This equipment decapsulates the CPS packets coming from the input ports (Terminal Adapters). Then, it encapsulates the CPS packets and sends them to the output port (i.e., the network). In the reverse direction, the inverse operations are performed.

As stated before, multiplexing is based on time scheduling, and the service discipline of each queue, corresponding to each input channel, is controlled by the scheduling algorithm of the Concentrator.

The maximum possible output bit rate is the sum of the bit rates of all input lines. However, it is expected that some statistical gain (traffic concentration) will be possible, since the Terminal Adapters will not usually generate traffic at full line rate. Thus, reducing the output bit rate will increase the efficiency. On the other hand, the higher is the output bit rate the lower is the delay introduced by the Concentrator. Dimensioning of the Concentrator scheduling algorithm should take into account this trade-off.

The transmission capacity and QoS guarantees (Service Category and traffic parameters) must be negotiated with the network operator [6]. Thus, transmission efficiency depends on the capacity assigned to the connection.

\section{SCHEDULING ALGORITHMS}

Scheduling Algorithms for the Terminal Adapter and Concentrator have been developed in order to perform traffic aggregation [7]. These algorithms must satisfy two main goals: guarantee a bounded delay for services with time critical requirements, and keep high transmission efficiency.

\section{A. Terminal Adapter Scheduling Algorithm}

This functional block, which belongs to the sending module of the Terminal Adapter, is responsible for scheduling the input information flows, taking into account the delay requirements of each service.

The parameters associated with each traffic class, which depend on the service characteristics, allow the implementation of a priority mechanism to efficiently serve the FIFOs associated with each traffic source. According to the traffic class, the scheduling algorithm will implement a priority mechanism in order to satisfy the delay requirements of each service, and at the same time optimizing the efficiency when assembling CPS packets. Thus, associated with each information flow there is a FIFO and a state table that keeps the following parameters:

- FIFO sizes in octets,

- Traffic class of each flow,

- Time-stamp of the FIFO oldest octet (octet at the head of the FIFO),
- For the MD class - Maximum delay that guarantees the correct delivery of the information.

Taking into account these parameters, the scheduler performs the following algorithm:

1. Calculates the service instants of the MD class FIFOs (deadlines), as a function of their maximum delays,

2. While these deadlines are not reached, sequentially serves the biggest FIFO (it can be of either class MD or D),

3. When the deadline of one of the MD class FIFOs is reached, it must be served,

4. If there is no information in the FIFOs of classes MD or D, it transmits the packets of the biggest FIFO of ME class.

Thus, while the service instants of the MD class packets are not reached, the priority criterion is based on the selection of the FIFO that has, in a given instant, the largest number of octets. The ME class FIFOs are served only when there is no information in the FIFOs of the remaining classes.

Fig. 4 illustrates the traffic scheduling mechanism, where the multiplexing structure of the AAL-2 CPS packets is used.

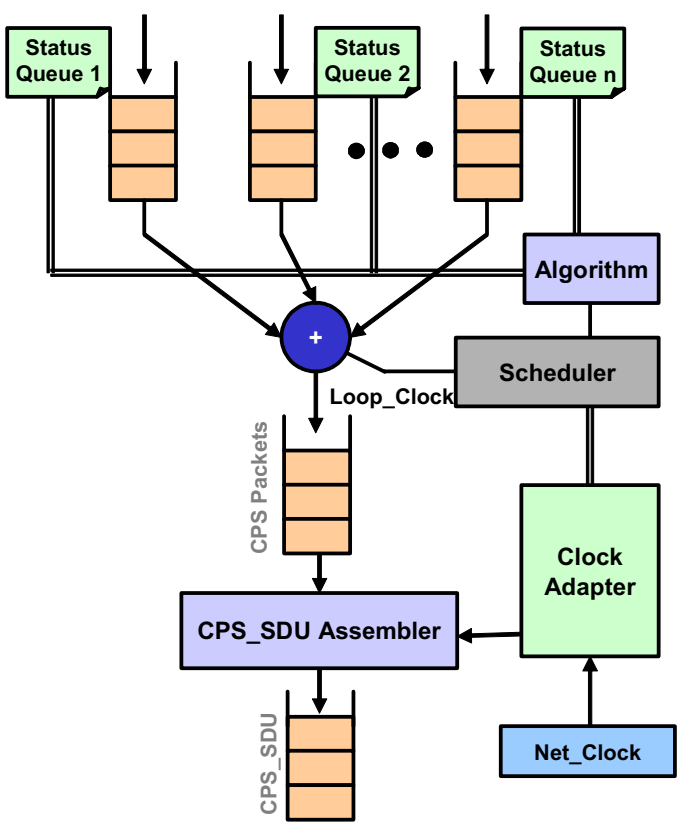

Fig. 4. Terminal Adapter traffic scheduling mechanism.

At each instant, the scheduler tries to read from the selected FIFO the maximum possible number of octets (45) in order to completely fill a CPS packet, thus keeping the overhead at the minimum. In case all FIFOs are empty, the scheduler will not assemble any CPS packet and the algorithm returns to the starting point.

Due to the fact that low bit rate flows of MD class may produce small amounts of information compared to the flows of other classes, and to the necessity of giving some priority to these services, it might not be possible to fill the corresponding CPS packets with the maximum size. In these cases the loss of efficiency, associated with the creation of smaller packets, can 
occur due to the need to satisfy the delay requirements of these services.

\section{B. Concentrator Scheduling Algorithm}

This functional block is responsible for scheduling CPS packets received from the Terminal Adapters. Its goal is to increase the final multiplexing efficiency without introducing a significant delay to the several data flows.

This module receives, at each input port, a CPS packet flow coming from Terminal Adapters. Besides the user information, each CPS packet has a header that identifies, among other things, the traffic class (UUI) and the packet size (LI). Since the implementation of a mechanism that takes into account the maximum delay associated to the MD class flows would be rather complex, the priority criterion, implemented by the scheduling algorithm, must minimize the queuing time of the packets of this class. In addition it achieves a higher multiplexing efficiency and thus allows reducing transmission costs due to bandwidth saving. Thus, the packets arriving at each port are directly routed to three queues, according to their traffic class. The algorithm serves in the first place the FIFOs of MD class packets, then the D class packets, and finally those of ME class. In this way, with the introduction of a small delay, it is possible to implement a mechanism that serves the MD class packets with highest priority and thus minimizes the delay associated with services of this class. Fig. 5 shows the Concentrator traffic scheduling mechanism.

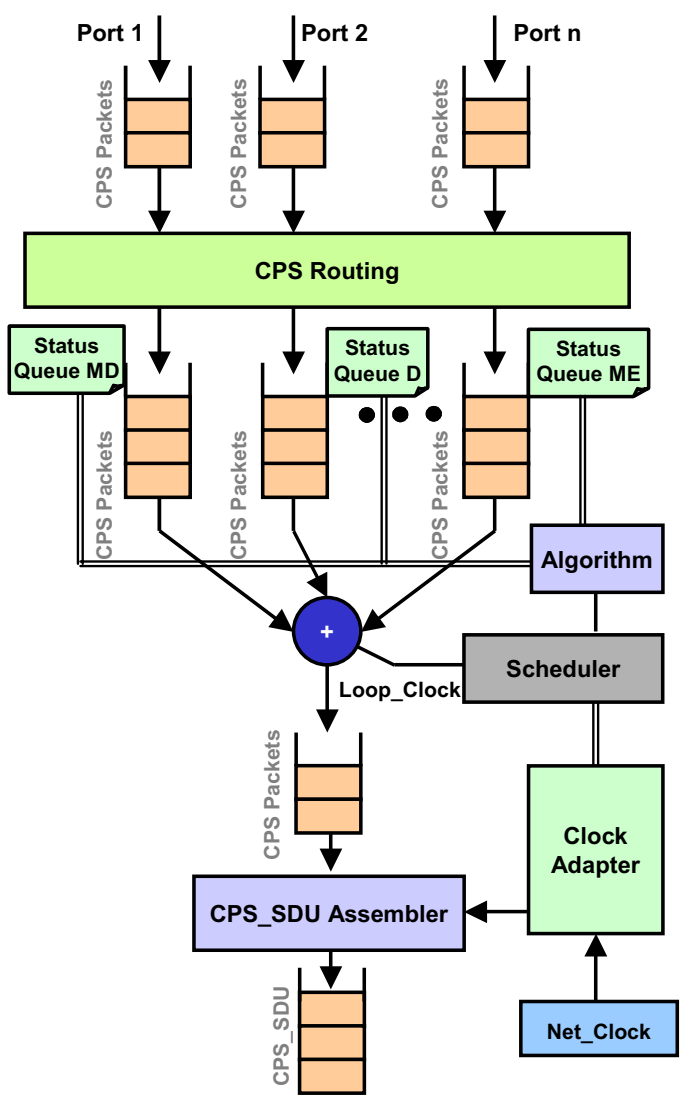

Fig. 5. Concentrator traffic scheduling mechanism.
The functional structure of this module is basically identical to the one used for the Terminal Adapter. This algorithm implements a simple priority mechanism, since it does not calculate the deadlines for sending the MD class packets. On the other hand, with this algorithm, it is necessary to process the LI fields of each packet, in order to determine its length. Thus, the scheduler will use the following algorithm:

1. At each scheduling instant, the MD class FIFO packets are processed,

2. In case the MD class FIFO is empty, the packets of D class FIFO are processed,

3. In case the D class FIFO is empty, the packets of the ME class FIFO are processed.

Due to the concentration effect, a ME class FIFO overflow may occur, since this FIFO has the lower service priority. In these situations, the algorithm will reject the packets that exceed the FIFO capacity.

\section{PERFORMANCE ANALISYS}

The system evaluation was based on a simplified test-bed developed for this purpose. It consists of a set of simulation programs written in C Language [4], which includes models of artificial sources that allowed the creation of specific test scenarios, difficult to obtain with real sources. Performance was evaluated in terms of:

- Multiplexing efficiency,

- Maximum delays and queue sizes of the different flows,

- Maximum bandwidth of the output aggregated flow.

The tests carried out for the Terminal Adapter were based on two concrete scenarios that were used to evaluate, respectively, the capacity of integrating flows associated with services with different delay requirements (Scenario-1), and the capacity to support a high number of low bit rate flows in an efficient way (Scenario-2).

Table II shows the characterization of sources used in both simulation scenarios.

TABLE II

SIMULATED TRAFFIC SOURCES

\begin{tabular}{|c|c|c|c|}
\hline Source & Bit rate (average) & Type & Class \\
\hline S1 - Sensor & $800 \mathrm{bit} / \mathrm{s}$ & Constant & MD \\
\hline S2 - Data & $16 \mathrm{kbit} / \mathrm{s}$ & Variable & D \\
\hline S3 - Actuator & $80 \mathrm{bit} / \mathrm{s}$ & Random & MD \\
\hline S4 -Voice & $16 \mathrm{kbit} / \mathrm{s}$ & Constant & MD \\
\hline S5 - Video & $\mathbf{8 0 \mathrm { kbit } / \mathrm { s }}$ & Variable & ME \\
\hline Total & $112 \mathrm{880} \mathrm{bit} / \mathrm{s}$ & & \\
\hline
\end{tabular}

MD class is assigned to Sensor, Actuator and Voice sources, D class to Data sources and ME class to Video sources. Each MD class flow will have an associated target delay as a function of its specific service characteristics. Voice sources are the most sensitive to delay, while Video sources load the system with the highest amount of data. 
Scenario-1 uses all Sources listed in Table II and Scenario-2 uses only Sensor (S1) and Data (S2) sources, since it is expected that these will be predominant in these kinds of applications. The target delays defined for S1, S3 and S4 flows were, respectively, $200 \mathrm{~ms}, 50 \mathrm{~ms}$ and $15 \mathrm{~ms}$.

\section{A. Simulation of the Terminal Adapter}

Table III shows some simulation results obtained with Terminal Adapter tests in scenario-1.

TABLE III

PERFORMANCE OF THE TERMINAL ADAPTER: SCENARIO-1.

\begin{tabular}{|c|c|c|c|c|c|c|}
\hline & \multicolumn{5}{|c|}{ Maximum Delay (ms) } & \\
\hline Bandwidth (kHz) & S1 & S2 & S3 & S4 & S5 & CPS \\
\hline $\mathbf{1 8 0 0}$ & 0.2 & 1.6 & 0.2 & 0.8 & 3.4 & 39.01 \\
\hline $\mathbf{3 6 0}$ & 1.0 & 9.0 & 1.0 & 4.2 & 21.9 & 39.10 \\
\hline $\mathbf{1 5 0}$ & 2.4 & 21.4 & 2.4 & 9.8 & 151.7 & 39.15 \\
\hline $\mathbf{1 2 0}$ & 3.0 & 29.9 & 3.0 & 14.8 & 632.4 & 39.17 \\
\hline
\end{tabular}

Each row of the table depicts simulation results for a specific bandwidth of the output aggregated flow, represented in the first column. Columns S1 to S5 indicate the maximum delays of the corresponding service flows of Table II.

Table III shows two important features of the Terminal Adapter behaviour:

- MD target delays were not overtaken,

- High multiplexing efficiency (CPS value).

Table IV shows results obtained in scenario-2.

TABLE IV

PERFORMANCE OF THE TERMINAL ADAPTER: SCENARIO-2.

\begin{tabular}{|c|c|c|c|c|}
\hline \multicolumn{2}{|c|}{} & \multicolumn{2}{|c|}{ Maximum Delay } & \\
\hline S2/S1 & Bandwidth (kHz) & S1 (ms) & S2 (ms) & CPS (bytes) \\
\hline $5 / 5$ & 87.8 & 20.5 & 2054 & 35.65 \\
\hline $10 / 5$ & 180 & 10.5 & 1047 & 41.11 \\
\hline $15 / 5$ & 257.1 & 7.0 & 691 & 43.67 \\
\hline $20 / 5$ & 327.3 & 5.5 & 964 & 45.17 \\
\hline
\end{tabular}

The first column of Table IV indicates the ratio between S1 and S2 sources. Since S2 sources (D-class) do not impose time constraints to the scheduler, the multiplexing efficiency (CPS) will increase with the $\mathrm{S} 2 / \mathrm{S} 1$ ratio.

On the other hand, MD target delay, associated with S1 sources, decreases when the number of S2 sources increases.

\section{B. Simulation of the Concentrator}

To evaluate the performance of the Concentrator it is necessary to quantify the delay of MD class flows, as a function of the MD input packet rate.

Table $\mathrm{V}$ shows some results, which put in evidence the following characteristic: the delay of MD class flows is quite small, except when these are the only flows at the Concentrator input ports.
TABLE V

PERFormance OF THE CONCENTRATOR.

\begin{tabular}{|c|c|c|c|}
\hline MD Sources (\%) & Delay $_{\text {MD }}(\mathbf{m s})$ & Delay $_{\mathbf{D}}(\mathbf{m s})$ & Delay $_{\text {ME }}(\mathbf{m s})$ \\
\hline 20 & 0.34 & 0.69 & 1.95 \\
\hline 60 & 0.34 & 1.17 & 3.35 \\
\hline 80 & 0.46 & 3.04 & 9.36 \\
\hline 100 & 27.7 & n.a & n.a \\
\hline
\end{tabular}

C. System Analysis

The simulations already carried out showed good performance of the proposed scheduling algorithms, both in terms of meeting the target delays of the input sources, and multiplexing efficiency.

Detailed simulation results, which will be published soon, confirmed the improvement of the global multiplexing efficiency, as expected. In the case of delay, it was verified that it depends on the value of the bandwidth allocated to the Concentrator, especially when most of the flows were not of the MD class. The contribution of the Concentrator to the total delay of MD Class flows is quite small, which allows concluding that adding a Concentrator to the architecture is very efficient. It allows a second aggregation level to the traffic handled by Terminal Adapters, thus reducing the wide area communications costs, at the expenses of a small delay penalty.

\section{CONCLUSIONS}

In this paper we have proposed and described a system architecture for aggregating low bit rate traffic in control applications. The use of AAL-2 protocol, as the supporting multiplexing structure, is appropriate to an efficient use of the bandwidth.

Simulation results for the Terminal Adapters showed good performance in terms of delay requirements and efficiency in supporting the aggregation of a high number of low bit rate flows.

As far as the Concentrator, it was verified that, in spite of the low complexity of the scheduling algorithm, it is equally efficient in improving the global multiplexing efficiency.

As a final conclusion, it can be stated that the proposed system architecture supports the establishment of remote connections among different data acquisition and control systems, in an efficient way and with QoS guarantees.

\section{REFERENCES}

[1] McDysan, D. E., Spohn, D. L., "ATM Theory and Application", McGraw-Hill Series on Computer Communications, 1995.

[2] C. Lu, B. Blum, T. Abdelzaher, J. Stankovic, and T. He, RAP; "A RealTime Communication Architecture for Large-Scale Wireless Sensor Networks"; Real-Time Technology and Applications Symposium; 2002.

[3] ITU-T, Rec. G.114, "One-way transmission time", May, 2000.

[4] Cabral, J.M.; "A System Architecture for Low Bit Rate Traffic Aggregation in Control Applications", PhD Thesis, University of Minho, 2005.

[5] ITU-T, Rec. I.363.2 - "B-ISDN ATM Adaptation Layer Specification: Type 2 AAL", September 1997.

[6] ATM Forum, AF-TM-0121.000, "Traffic Management Specification Version 4.1", 1999

[7] Keshav, S.; "An Engineering Approach to Computer Networking", pp. 209-246 Addison-Wesley, 1997. 